\title{
Rhizoma Drynariae promotes the Osteogenic differentiation of Bone Mesenchymal Stem Cells by activating the Wnt/ $\beta$-catenin Signaling Pathway
}

\section{Yi-Wei Shen}

Heilongjiang University of Chinese Medicine

Yi Li

Northeast Normal University

Zuo Li

Northeast Normal University

Bing-you Yang

Northeast Normal University

Xue Li ( $\nabla$ yellwell@sina.com )

Heilongjiang University of Chinese Medicine https://orcid.org/0000-0002-2167-0228

\section{Research}

Keywords: Rhizoma Drynariae, osteogenic differentiation, bone mesenchymal stem cells, Wnt/ $\beta$-catenin signaling pathway

Posted Date: June 3rd, 2021

DOI: https://doi.org/10.21203/rs.3.rs-571947/v1

License: (1) This work is licensed under a Creative Commons Attribution 4.0 International License. Read Full License 


\section{Abstract}

Background: Rhizoma Drynariae (RD), a traditional Chinese medicine with pleiotropic biological activities, exerts a protective effect against age-related osteoporosis. Osteoporosis is a serious clinical problem and characterized by the deterioration in bone volume and strength, mainly due to the dysfunction of bone marrow stromal cells (BMSCs). However, it remains unclear whether RD-containing serum regulates the osteogenic differentiation of BMSCs via Wnt/ $\beta$-catenin signaling pathway.

Methods: The effects of RD-containing serum on the osteogenesis of BMSCs were detected via quantitative reverse transcriptase-polymerase chain reaction (qRT-PCR), western blotting, alkaline phosphatase (ALP) activity assay and alizarin red staining. Using Dickkopf-related protein-1 (DKK1), an inhibitor of the Wnt/ $\beta$-catenin signaling pathway, we examined whether RD-containing serum regulates osteoblast differentiation of BMSCs via the Wnt/b-catenin signalling pathway.

Results: The results showed that RD-containing serum promoted the BMSC proliferation and ALP activities, as well as up-regulated the expression of osteogenic markers and Wnt/ $\beta$-catenin pathwayrelated genes, i.e., runt-related transcription factor 2 , Sp7, osteocalcin, $\beta$-catenin and Wnt3a, in BMSCs. Moreover, we found that RD-containing serum activated the Wnt/ $\beta$-catenin pathway. Adding DKK1 to the RD-containing serum could decrease the promotion of osteogenic differentiation of RD-containing serum on BMSCs.

Conclusions: Collectively, RD-containing serum could promote the osteogenic differentiation of BMSCs, and the potential mechanism may involve regulation of Wnt/ $\beta$-catenin signaling.

\section{Introduction}

Osteoporosis (OP) is a metabolic bone disease involving low bone mineral density (BMD), which usually leads to lower bone resistance to load and fragility fracture, and its development is affected by imbalance of bone homeostasis, with excessive bone resorption compared with bone formation ${ }^{1-4}$. OP is characterized by decreased bone mass and microarchitectural deterioration with a subsequent increased risk of fragility fracture effects. Multiple agents exist that are effective for the treatment of osteoporosis, such as teriparatide, bisphosphonates and denosumab ${ }^{3,5}$. Teriparatide has proven effective in reducing both vertebral and non-vertebral fractures and is currently the only bone formation-accelerating drug, whereas bisphosphonates act mainly through inhibition of bone resorption. However, teriparatide has been recently listed and its safety has not been fully verified ${ }^{6}$. Studies have shown that the oral bisphosphonate route is associated with a high incidence of osteonecrosis of the jaw and other complications ${ }^{4,7}$. Phytochemicals, such as flavonoids, with therapeutic and preventive effects against OP have recently received more and more attention because they are potentially more suitable for long-term use than traditional therapeutic chemical compounds, such as denosumab, teriparatide, abaloparatide, romosozumab, and selective estrogen receptor modulators ${ }^{1}$. 
The Wnt/ $\beta$-catenin signaling pathway, which regulates the expression of osteogenic differentiation marker genes and the dissociation of physiological bone remodeling stages, has a beneficial role in bone formation and thus has been identified as a potential target for OP treatment ${ }^{8}$. So far, three different WNT signaling pathways have been described, all of which are activated by binding of the WNT ligands to the Frizzled receptors. Phytochemicals, mechanical stimuli and extracellular Wnt proteins could activate the Wnt/ $\beta$-catenin signaling pathway and promote bone formation ${ }^{9}$. DKK1, an inhibitor of the Wnt/ $\beta$-catenin signaling pathway, can competitively bind to LRP $5 / 6$ and thus has been identified as an inhibitor of the Wnt/ $\beta$-catenin signaling pathway ${ }^{10}$.

Rhizoma Drynariae (RD), the dried rhizome of Drynaria fortune (Kunze) J. Sm., has been widespread and been proved to be effective in treating OP, bone fractures and defects ${ }^{11-16}$. Due to the minimum side effects and favorable cost-effective therapeutic effects, RD has been widely used in clinical practices for more than 2,100 years in China. RD, as one of the most common clinically used folk medicines, has the effects of tonifying kidney, promoting blood circulation, stopping bleeding, and prolonging damage. its main chemical components are flavonoids, triterpenes, phenylpropanoids, etc. ${ }^{16,17}$. RD has been proved to exert multifaceted pharmacological effects on the bone homeostasis, such as up-regulating the expression levels of BMP-2 and Runx $2^{18,19}$, promoting osteoblast proliferation and differentiation ${ }^{20,21}$, inhibiting osteoclast activity and preventing bone loss ${ }^{17,22,23}$. In recent years, there have been a lot of reports on RD's anti-osteoporosis treatment ${ }^{14,17,23}$, but the mechanism of its anti-osteoporosis action from the perspective of $W n t / \beta$-catenin signaling pathway is still very few.

In the current study, we used RD-containing serum to stimulate the osteogenic differentiation of bone marrow mesenchymal stromal cells (BMSCs) and used DKK1 as the inhibitor of Wnt/ $\beta$-catenin to explore the osteogenesis of BMSCs and the potential mechanisms of RD.

\section{Materials And Methods}

\section{Preparation of ethanol extracts of RD}

Rhizoma Drynariae (collection in Hunan, China) was purchased from pharmacy department of Second Affiliated Hospital of Heilongjiang University of Chinese Medicine (Heilongjiang, Harbin, China), and authenticated as the dried rhizome of Drynaria fortunei (Kunze) J. Sm. by Professor Bingyou Yang (School of Chinese Medicine, Heilongjiang University of Chinese Medicine, China). Content of naringin (pharmacological active component in Rhizoma Drynariae, $0.84 \%$ ) was determined referring to the authentic method (pharmacopeia of the People's Republic of China 2010, volume I, page 239). Powdered $\mathrm{RD}(500 \mathrm{~g})$ was extracted with $75 \%$ ethanol $(6000 \mathrm{~mL}, 3 \times)$ under thermal reflux for $2.5 \mathrm{~h}$. After filtration, the ethanol extract was concentrated under reduced pressure. The residue was dissolved in $0.5 \%$ sodium carboxyl methyl cellulose to give an extract with a concentration of $2 \mathrm{~g} / \mathrm{mL}$ (expressed as the weight of raw materials).

\section{Animal experiments}


Ten female Sprague-Dawley (SD) rats were purchased from the Animal Experiment Center of Heilongjiang University of Chinese Medicine (permit number: SYXK-2016004) and were housed at Heilongjiang University of Chinese Medicine (Heilongjiang, Harbin, China). This experiment was approved by the Animal Ethics Committee of the Second Affiliated Hospital of Heilongjiang University of Chinese Medicine. All animal procedures were carried out in accordance with the UK Animals (Scientific Procedures) Act 1986. The rats were maintained under standard laboratory conditions (temperature of $21-23{ }^{\circ} \mathrm{C}$, relative humidity of $45-65 \%$, and $12 \mathrm{~h} / 12 \mathrm{~h}$ light/dark cycle) with food and water freely available.

The 32 rats were randomly divided into three groups: high RD group $(n=10)$ in which RD extract was administered orally at dose of $40 \mathrm{mg} / \mathrm{kg}$ body weight once daily between 8:00 and 10:00 a.m. for the following 7 days; low RD group $(n=10)$ RD extract was administered orally at dose of $20 \mathrm{mg} / \mathrm{kg}$; control group $(n=12)$ which was fed naturally without any handle for following 7 days.

\section{Preparation of RD-containing serum}

At $1 \mathrm{~h}$ after the last gavage, SD rats were anesthetized with chloral hydrate. With a sterilized technique, blood was collected through their abdominal aorta and centrifuged at $1,610 \times \mathrm{g}$ for $10 \mathrm{~min}$ to separate the drug-containing serum. The same group of serum was mixed with each other. Then the serum was inactivated at $56^{\circ} \mathrm{C}$ for $30 \mathrm{~min}$ in water bath, filtered through a $0.22 \mathrm{~mm}$ pore size filter, dispensed into a cryotube, and stored in a $-80^{\circ} \mathrm{C}$ refrigerator. Mixing, filtration, and dispensing were all performed in a biosafety cabinet.

\section{Chemicals and reagents}

RIPA lysis buffer, BCA kit, Fetal bovine serum (FBS), a-minimum essential medium (a-MEM), penicillin and streptomycin were purchased from Beyotime (Beyotime, Beijing, China). The recombinant human Dickkopf-related protein 1 (DKK1) was purchased from MCE (Shanghai, China). The alkaline phosphatase activity measurement kit was purchased from Sigma Company (Sigma-Aldrich, USA). Antibodies to $\beta$-catenin, Wnt7a, Wnt3a, Sp7, Runx2 and $\beta$-actin and appropriate secondary antibodies were obtained from Abcam (CA, USA). Reverse transcription system and GoTaq ${ }^{\circledR}$ 2- Step RT-qPCR system were obtained from Promega (Madison, Wisconsin, USA).

\section{Isolation and culture of BMSCs}

BMSCs were obtained from the bone marrow of the femurs and tibias of 3-week-old rats according to our previously reported study ${ }^{24}$. Briefly, the bone marrow was flushed out with $2 \mathrm{ml}$ of a-MEM containing $10 \%$ FBS using a $5-\mathrm{ml}$ syringe. The bone marrow was layered on top of the separating medium. After cell centrifugation at a speed of $3500 \mathrm{r} / \mathrm{min}$, the buffy coat was removed and seeded in a culture flask. The isolated BMSCs were cultured in a-Minimal Essential Medium with $10 \%(\mathrm{v} / \mathrm{v}) \mathrm{FBS}$ and $1 \%(\mathrm{v} / \mathrm{v})$ penicillin and streptomycin in a $5 \% \mathrm{CO}_{2}$ incubator at $37^{\circ} \mathrm{C}$. The BMSCs from 3 to 5 passages were used for subsequent experiments. Osteogenic induction medium (OIM) comprised the basal culture medium 
supplemented with $5 \times 10^{-5} \mathrm{M} \mathrm{L-ascorbic} \mathrm{acid,} 10^{-2} \mathrm{M} \beta$-sodium glycerophosphate, and $10^{-7} \mathrm{M}$ dexamethasone.

\section{Cell culture and treatments}

The experiment was performed as follows: (1) The BMSCs were treated with a-MEM supplemented with $10 \%$ FBS and $1 \%$ high or low RD-containing serum. The control BMSCs were treated with a-MEM supplemented with $10 \%$ FBS and $1 \%$ serum without RD-containing. (2) The BMSCs were treated with OIM supplemented with $10 \%$ FBS and $1 \%$ high or low RD-containing serum. The control BMSCs were treated with OIM with $10 \%$ FBS and $1 \%$ serum without RD-containing. To determine the potential effects of the Wnt/ $\beta$-catenin signaling pathway on the osteogenic differentiation of BMSCs, we used DKK1 (0.6 $\mathrm{mg} / \mathrm{mL}$ ) to inhibit the $\mathrm{Wnt} / \beta$-catenin signaling.

\section{Cell proliferation assay}

The MTT assay was performed to evaluate the effect of RD-containing serum on the proliferation of BMSCs. BMSCs were plated at a density of $1 \times 10^{4}$ per well in 96-well plates and incubated in a-MEM for $20 \mathrm{~h}$. When BMSCs covered the 96-well plates, the RD-containing serum was added for 1, 2, 4, and 8 days. At days $1,2,4$, and 8 , MTT $(5 \mathrm{mg} / \mathrm{mL})$ was added to the a-MEM medium. Then, $100 \mu$ l of dimethyl sulfoxide (DMSO) added to the 96-well to dissolve the formazan crystals and and the absorbance at 570 nm was measured using a microplate reader (SpectraMax Plus384, Molecular Devices, Sunnyvale, CA, USA).

\section{ALP activity assay}

For quantitative ALP activity determination, the cells were lysed in $0.5 \%(\mathrm{v} / \mathrm{v})$ Triton X-100 in PBS for 30 min. ALP activity was measured by the conversion of the $\mathrm{p}$-nitrophenyl phosphate liquid substrate system (Sigma-Aldrich). The absorbance was measured at $405 \mathrm{~nm}$ (Bio-Rad, Hercules, CA, USA). The same cell lysate was used to determine the protein content with the BCA kit according to the manufacturer's instructions.

\section{Quantitative reverse transcriptase-polymerase chain reaction (qRT-PCR)}

Total RNA was extracted using the TRIzol® reagent (Invitrogen), and RNA concentrations were measured with a NanoDrop ${ }^{\circledR} 8000$ spectrophotometer (Thermo Fisher Scientific, Waltham, MA, USA). RNA was reverse-transcribed to cDNA using a GoScript ${ }^{\mathrm{TM}}$ Reverse Transcription System. The level of mRNA expression was quantified via qRT-PCR using a GoTaq ${ }^{\circledR}$ 2-Step RT-qPCR System and a CFX96TM realtime system (Bio-Rad, Hercules, CA, USA). The primer sequences are shown in Table 1. We used $\beta$-actin as the reference control. The relative mRNA levels of Runx2, Sp7, $\beta$-catenin, Wnt3a, Wnt7a, c-myc, cyclin-D, Tcf7, c-jun, and Lef-1 were expressed as fold changes normalized to GAPDH mRNA and were analyzed according to the $2^{-\Delta \Delta C t}$ comparative method.

\section{Western blot analysis}


BMSCs were lysed using the RIPA buffer containing phenylmethane sulfonyl fluoride (PMSF). Protein concentrations were quantified by the BCA assay. Proteins from individual groups $(100 \mu \mathrm{g})$ were separated in a 10\% sodium dodecyl sulfate-polyacrylamide gel and transferred to an Immobilon PVDF membrane (Millipore, Bedford, MA, USA). The membrane was blocked with $5 \%$ nonfat dry milk in PBST for $2.5 \mathrm{~h}$ at room temperature and then incubated with rabbit polyclonal anti-rat $\beta$-catenin, Runx2, Sp7, Wnt4a and Wnt7a antibodies for $2 \mathrm{~h}$ at room temperature. After washing three times for $10 \mathrm{~min}$ each with PBST, the PVDF membranes were incubated with the corresponding secondary antibodies at room temperature for $2.5 \mathrm{~h}$ and washed three times with PBST. The immunoreactive bands from triplicates were visualized and quantified using the Odyssey Infrared Imaging System (LI-COR, Lincoln, NE, USA).

\section{Immunofluorescence staining}

At day 8 of cell culture, cells were fixed with $4 \%$ paraformaldehyde for ten minutes and washed with PBS for three times. Then, $0.15 \%$ Triton X-100 was added for 15 min to break the cell membranes. Next, the BMSCs were blocked with $1 \%$ bovine serum albumin (BSA) to prevent nonspecific reactions. BMSCs were then incubated with anti- $\beta$-catenin antibodies (Abcam, USA, 1:500) overnight in a $4{ }^{\circ} \mathrm{C}$ refrigerator.

BMSCs were placed at room temperature for $1 \mathrm{~h}$ and washed with PBS three times. BMSCs were then incubated with the fluorescein isothiocyanate (FITC)-linked rabbit anti-mouse IgG antibody and diamidino-2-phenylindole (DAPI) for $2 \mathrm{~h}$. Then, images of BMSCs were taken using a confocal microscope (Zeiss, Germany).

Table 1. The gene sequence of the Runx2, Sp7, $\beta$-catenin, Wnt3a, Wnt7a, c-myc, cyclin-D, Tcf7, c-jun, and Lef1. F, forward, R, reverse.

\begin{tabular}{|lll|}
\hline Gene & F & R \\
Runx2 & GACTGTGGTTACCGTCATGGC & ACTTGGTTTTTCATAACAGCGGA \\
Sp7 & ACCTCTTGAGAGGAGACGGG & CTGTTGAGTCTCGCAGAGGG \\
B-catenin & ATAGTTGAAGGGTGCACTGGG & GTCGGTATCAAACCAGGCCA \\
Wnt3a & GGCTCCTCTCGGATACCTCT & ACAGAGAATGGGCTGAGTGC \\
Wnt7a & GATGCCCGAGAGATCAAGCA & CTGTGAGTATCCAGGCAGGG \\
c-myc & TGAAAAGAGCTCCTCGCGTT & TTCTCGGAGACCAGTTTGGC \\
cyclin-D & CTACCGCACAACGCACTTTC & GCCAAAGCTGTGCCTTTTCA \\
\hline Tcf7 & AAGGAGTTCACAGACCGCTG & ATCTGGTTCCACGTGAGCTG \\
c-jun & CCAACCAACGTGAGTGCAAG & GAGGGCATCGTCGTAGAAGG \\
Lef1 & CTGTCAGGCGACACTTCCAT & CTGCGCTCTCCTTTAGCGTA \\
GADPH & GTGAAGGTCGGTGTGAACGG & CCCCATTTGATGTTAGCGGG \\
\hline
\end{tabular}




\section{Results}

\section{MTT assay analysis of the BMSC proliferation}

The MTT assay showed that high RD-containing serum significantly enhanced proliferation of BMSCs at days 2,4 , and 8 compared with the control group $(P<0.05)$. However, low RD-containing serum significantly enhanced proliferation of BMSCs at days 4 , and 8 compared with the control group $(\mathrm{P}<$ 0.05). The effect of high RD-containing was more pronounced than that of low RD-containing serum $(P<$ 0.05). Compared with the high RD-containing serum-treated group, BMSCs co-cultured with DKK1 (0.6 $\mathrm{mg} / \mathrm{mL}$ ) showed a significant decrease in proliferation $(P<0.05)$. Compared with control group, the proliferation of BMSCs in DKK1 $(0.6 \mathrm{mg} / \mathrm{mL})$ group was increased, however, the difference was not statistically significant $(P>0.05$, Fig. 1$)$.

\section{ALP activity assay of the BMSC osteogenic differentiation}

The ALP activity results shown that the high and low RD-containing serum-treated groups significantly increased the ALP activity at days 4, 8 and 16 compared with the control group $(P<0.05)$. The effect of high RD-containing was more pronounced than that of low RD-containing serum $(P<0.05)$. When DKK1 $(0.6 \mathrm{mg} / \mathrm{mL})$ was added in the high RD-containing serum-treated group, the ALP activity decreased with statistical significance $(P<0.05$, Fig. 2).

\section{Alizarin red staining (ARS) results}

BMSCs were cultured in an OIM for 16 days, fixed and stained with Alizarin red. Both low and high RDcontaining serum markedly increased the osteogenic differentiation of BMSCs, while DKK1 inhibited the promotion of high RD-containing serum on the osteogenic differentiation of BMSCs (Fig. 3).

\section{qRT-PCR results}

The PCR results indicated that, compared with the control group, both high and low RD-containing serum increase in the expression of the osteogenic genes (Runx2, Sp7 and Ocn) and the Wnt/ $\beta$-catenin pathwayrelated genes ( $\beta$-catenin, Wnt3a, Fig. 4). The effect of high RD-containing was more pronounced than that of low RD-containing serum $(P<0.05)$. When DKK1 $(0.6 \mathrm{mg} / \mathrm{mL})$ was added in the high RD-containing serum-treated group, the relative expression of these genes decreased significantly $(P<0.05$, Fig. 4$)$. However, no significant difference was observed for the reference gene Wnt7a $(P>0.05$, Fig. 4).

Furthermore, the relative expression of c-myc, cyclin-D, Tcf7, c-jun, and Lef1 in the high and low RDcontaining serum-treated groups was statistically significantly higher than in the control group $(P<0.05$, Fig. 5). When DKK1 $(0.6 \mathrm{mg} / \mathrm{mL})$ was added in the high RD-containing serum-treated group, the relative expression of these genes decreased significantly $(P<0.05$, Fig. 5).

\section{Western blot results}


As shown in Fig. 6, the high RD-containing serum upregulated the expression of the osteogenic genes (Runx2, and Sp7) and the Wnt/ $\beta$-catenin pathway-related genes ( $\beta$-catenin, and Wnt3a). There was no significant difference between the expression of Wnt7a in the experimental and control groups. When DKK1 $(0.6 \mathrm{mg} / \mathrm{mL})$ was added in the high RD-containing serum-treated group, the relative expression of these genes decreased significantly $(P<0.05)$. However, no significant difference was observed for the reference gene Wnt7a $(P>0.05)$.

\section{RD-containing serum induced $\beta$-catenin nuclear translocation in BMSCs}

Next, we examined whether the RD-containing serum could activate Wnt/ $\beta$-catenin signaling and enhance $\beta$-catenin translocation to the nucleus. As shown in Fig. 7, when high RD-containing serum was added to the BMSCs, we observed the $\beta$-catenin (green) transfer into the nucleus (blue). The added DKK1 (0.6 $\mathrm{mg} / \mathrm{mL}$ ) blocked the RD-containing serum induced the nuclear translocation of $\beta$-catenin.

\section{Discussion}

Osteoporosis is a serious clinical problem and characterized by the deterioration in bone volume and strength, partly due to the dysfunction of BMSCs. This study indicates that RD-containing serum could enhance the proliferation of BMSCs in a concentration- and time-dependent manner. Furthermore, RDcontaining serum increases the osteogenic differentiation of BMSCs, which is reflected in the upregulation of ALP activity and increased number of calcified nodules in the RD-containing serum group. We have also found that the RD-containing serum promotes the expression of osteogenic genes (Runx2, Sp7, Ocn), and Wnt/ $\beta$-catenin-related genes (Wnt3a and $\beta$-catenin). Previous studies have identified the Wnt/ $\beta$-catenin signaling pathway as a mediator of BMSC differentiation to osteoblasts ${ }^{25-}$ 28. When the Wnt/ $\beta$-catenin inhibitor DKK1 is added $(0.6 \mu \mathrm{g} / \mathrm{mL})$ to the RD-containing serum, it negatively regulates osteogenic differentiation of BMSCs.

Research has shown that RD could enhance angiogenic-osteogenic coupling during distraction osteogenesis by promoting type $\mathrm{H}$ vessel formation through PDGF-BB/PDGFR- $\beta$ signaling pathway ${ }^{14}$. RD could promote osteoblast differentiation and mineralization in MC3T3-E1 cells through regulation of bone morphogenetic protein-2, alkaline phosphatase, type I collagen and collagenase- $1^{29}$. UPLC-MS metabolomics method can provide valuable insights into the effect and underlying mechanisms of RD protecting osteoporosis, and the results showed RD mainly plays a role in treating OP through lipid metabolic pathways, arachidonic acid metabolism and glycerol 3-phosphate ester metabolism ${ }^{12}$.Studies, based on network pharmacology, showed that RD may play an anti-osteoporotic role by directly or indirectly targeting PI3k-akt signaling pathway, osteoclast differentiation, Wnt signaling pathway, and estrogen signaling pathway ${ }^{30}$. Cathepsin $\mathrm{K}$, which plays an important role in bone resorption and acts as a potential target in the treatment of osteoporosis, can be regulated by $\mathrm{RD}^{31}$. $\mathrm{RD}$ extract prevented the intracellular maturation of cathepsin $\mathrm{K}$ and thus, it was considered that RD is a pro-drug of a potent bone resorption inhibitor ${ }^{29}$. DR has a variety of effects on bone homeostasis through the promotion of 
osteoblastic differentiation and inhibition of osteoclast activity to prevent bone loss ${ }^{17,22,23}$. This study indicated that RD-containing serum could enhance the osteogenesis of BMSCs through the Wnt/ $\beta$ catenin signaling pathway.

Further, RD-containing serum could enhance the BMSC proliferation and ALP

activity, with the higher RD dose exerting a more pronounced effect than the lower RD dose. Next, we measured the mRNA and protein levels in the RD-containing serum-treated and control groups and showed that RD-containing serum could enhance the mRNA and protein levels of Runx2, Sp7, Wnt3a and $\beta$-catenin. Upon DKK1 addition to high RD-containing serum-treated medium, the relative levels of Runx2, $\mathrm{Sp7}$, Wnt3a and $\beta$-catenin were decreased.

To further analyze the the mechanism of Wnt / $\beta$-catenin signaling pathway, we tested whether RDcontaining serum enhanced the osteogenesis of BMSCs via activating Wnt/ $\beta$-catenin signaling. Wnt $/ \beta$ catenin signaling is a key regulator of bone biology and participates in the differentiation of BMSCs into osteoblasts. However, whether the RD-containing serum could activate Wnt/ $\beta$-catenin signaling and promote the osteogenic of BMSCs has been unknown. When BMSCs were cultured in the presence of both DKK1 and RD-containing serum, the relative expression of Runx2 and Sp7 was decreased. Thus, RDcontaining serum could enhance the osteogenesis of BMSCs into osteoblasts by regulating the $\mathrm{Wnt} / \mathrm{B}-$ catenin signaling pathway. We further explored the relative expression of Wnt3a, $\beta$-catenin, and Wnt7a and showed that RD-containing serum mainly acts through the regulation of Wnt3a rather than Wnt7a. $\beta$ catenin plays a critical role in the Wnt signaling pathway to facilitate downstream effects on gene expression $^{32}$. We used the immunofluorescence method to visualize the $\beta$-catenin translocation into the cell nucleus. Our results showed that RD-containing serum could enhance the $\beta$-catenin nuclear translocation, while DKK1 could inhibit the promotion of $\beta$-catenin nuclear translocation induced by RDcontaining serum.

In conclusion, RD-containing serum exerts a beneficial role in promoting the proliferation of BMSCs and osteogenic activity by increasing the expression of Runx2 and Sp7. Furthermore, RD-containing serum activates the $\mathrm{Wnt} / \beta$-catenin signaling pathway and increases osteogenic activity. Therefore, our findings indicate that RD-containing serum could enhance the osteogenesis of BMSCs through the Wnt/ $\beta$-catenin signaling pathway.

\section{Abbreviations}

RD: Rhizoma Drynariae; OP: osteoporosis; BMD: bone mineral density; BMSCs: bone marrow stromal cells; qRT-PCR: quantitative reverse transcriptase-polymerase chain reaction; ALP: alkaline phosphatase; ARS: alizarin red staining; DKK1: Dickkopf-related protein-1; Runx2: runt-related transcription factor 2; Ocn: osteocalcin; FBS: fetal bovine serum; a-MEM: a-minimum essential medium; OIM: osteogenic induction medium. 


\section{Declarations}

Acknowledgements

None.

\section{Authors' Contributions}

Yi-Wei Shen and Xue Li: Research design; Yi-Wei Shen, Xue Li, Yi Li, Bing-you Yang, and Zuo Li: Performance of experiments; Yi-Wei Shen, Xue Li, and Yi Li: Data analysis; Yi-Wei Shen and Xue Li: Manuscript preparation; Yi-Wei Shen, Yi Li, Bing-you Yang, and Xue Li: Manuscript revision; all authors approved the final manuscript as submitted.

\section{Funding}

This study was jointly funded by the National Natural Science Foundation of China (nos. 81904222), the Heilongjiang University of Traditional Chinese Medicine National Project Support (nos. 2019PT08), the Postdoctoral Program of Heilongjiang Province (LBH-Z20034), and the Research Fund Project of Heilongjiang University of Traditional Chinese Medicine (no. 201811).

\section{Availability of data and materials}

All data generated or analyzed during this study are included in this published article.

\section{Ethics approval and consent to participate}

This experiment was approved by the Animal Ethics Committee of the Second Affiliated Hospital of Heilongjiang University of Chinese Medicine. All animal procedures were carried out in accordance with the UK Animals (Scientific Procedures) Act 1986.

\section{Consent for publication}

Not applicable.

\section{Competing interests}

The authors declare that they have no competing interests.

\section{References}

1. Bellavia D, Dimarco E, Costa V, Carina V, De Luca A, Raimondi L, et al. Flavonoids in Bone Erosive Diseases: Perspectives in Osteoporosis Treatment. Trends Endocrinol Metab. 2021;32(2):76-94. doi:10.1016/j.tem.2020.11.007.

2. Chen X, Zhu X, Wei A, Chen F, Gao Q, Lu K, et al. Nrf2 epigenetic derepression induced by running exercise protects against osteoporosis. Bone research. 2021;9(1):15. doi:10.1038/s41413-020- 
00128-8.

3. Fontalis A, Kenanidis E, Prousali E, Potoupnis M, Tsiridis E. Safety and efficacy of denosumab in osteoporotic patients previously treated with other medications: a systematic review and metaanalysis. Exp Opin Drug Saf. 2018;17(4):413-28. doi:10.1080/14740338.2018.1430764.

4. Johnston CB, Dagar M. Osteoporosis in Older Adults. Med Clin N Am. 2020;104(5):873-84. doi:10.1016/j.mcna.2020.06.004.

5. Saul D, Drake MT. Update on Approved Osteoporosis Therapies Including Combination and Sequential Use of Agents. Endocrinol Metab Clin North Am. 2021;50(2):179-91. doi:10.1016/j.ecl.2021.03.010.

6. Hauser B, Alonso N, Riches PL. Review of Current Real-World Experience with Teriparatide as Treatment of Osteoporosis in Different Patient Groups. Journal of clinical medicine 2021; 10 (7): doi:10.3390/jcm10071403.

7. Hung C, Muñoz M, Shibli-Rahhal A. Anorexia Nervosa and Osteoporosis. Calcif Tissue Int. 2021. doi:10.1007/s00223-021-00826-3.

8. Elseweidy MM, El-Swefy SE, Shaheen MA, Baraka NM, Hammad SK. Effect of resveratrol and mesenchymal stem cell monotherapy and combined treatment in management of osteoporosis in ovariectomized rats: Role of SIRT1/FOX03a and Wnt/ $\beta$-catenin pathways. Arch Biochem Biophys. 2021;703:108856. doi:10.1016/j.abb.2021.108856.

9. Wang F, Tu P, Zeng K, Jiang Y. Total glycosides and polysaccharides of Cistanche deserticola prevent osteoporosis by activating Wnt/ $\beta$-catenin signaling pathway in SAMP6 mice. J Ethnopharmacol. 2021;271:113899. doi:10.1016/j.jep.2021.113899.

10. Ai M, Holmen SL, Van Hul W, Williams BO, Warman ML. Reduced affinity to and inhibition by DKK1 form a common mechanism by which high bone mass-associated missense mutations in LRP5 affect canonical Wnt signaling. Molecular cellular biology. 2005;25(12):4946-55. doi:10.1128/mcb.25.12.4946-4955.2005.

11. Huang J, Tong XF, Yu ZW, Hu YP, Zhang L, Liu Y, et al. Dietary supplementation of total flavonoids from Rhizoma Drynariae improves bone health in older caged laying hens. Poultry science. 2020;99(10):5047-54. doi:10.1016/j.psj.2020.06.057.

12. Jiang YC, Li YF, Zhou L, Zhang DP. UPLC-MS metabolomics method provides valuable insights into the effect and underlying mechanisms of Rhizoma Drynariae protecting osteoporosis. Journal of chromatography B, Analytical technologies in the biomedical and life sciences 2020; 1152122262. doi: 10.1016/j.jchromb.2020.122262.

13. Mu P, Hu Y, Ma X, Shi J, Zhong Z, Huang L. Total flavonoids of Rhizoma Drynariae combined with calcium attenuate osteoporosis by reducing reactive oxygen species generation. Experimental therapeutic medicine. 2021;21(6):618. doi:10.3892/etm.2021.10050.

14. Shen Z, Chen Z, Li Z, Zhang Y, Jiang T, Lin H, et al. Total Flavonoids of Rhizoma Drynariae Enhances Angiogenic-Osteogenic Coupling During Distraction Osteogenesis by Promoting Type H Vessel 
Formation Through PDGF-BB/PDGFR- $\beta$ Instead of HIF-1a/ VEGF Axis. Frontiers in pharmacology 2020; 11 503524. doi: 10.3389/fphar.2020.503524.

15. Sun X, Wei B, Peng Z, Chen X, Fu Q, Wang C, et al. A polysaccharide from the dried rhizome of Drynaria fortunei (Kunze) J. Sm. prevents ovariectomized (OVX)-induced osteoporosis in rats. J Cell Mol Med. 2020;24(6):3692-700. doi:10.1111/jcmm.15072.

16. Kang SN, Lee JS, Park JH, Cho JH, Park JH, Cho KK, et al. In vitro anti-osteoporosis properties of diverse Korean Drynariae rhizoma phenolic extracts. Nutrients. 2014;6(4):1737-51. doi:10.3390/nu6041737.

17. Wu L, Ling Z, Feng X, Mao C, Xu Z. Herb Medicines against Osteoporosis: Active Compounds \& Relevant Biological Mechanisms. Current topics in medicinal chemistry. 2017;17(15):1670-91. doi:10.2174/1568026617666161116141033.

18. Yao W, Zhang H, Fakhar EAKM, Ding Y, Waqas $M$, Mehmood K, et al. Effect of total flavonoids of Rhizoma Drynariae in thiram induced cytotoxicity of chondrocyte via BMP-2/Runx2 and IHH/PTHrP expressions. Ecotoxicology and environmental safety 2020; 206 111194. doi:

10.1016/j.ecoenv.2020.111194.

19. Yao W, Zhang H, Jiang X, Mehmood K, lqbal M, Li A, et al. Effect of Total Flavonoids of Rhizoma drynariae on Tibial Dyschondroplasia by Regulating BMP-2 and Runx2 Expression in Chickens. Frontiers in pharmacology 2018; 9 1251. doi: 10.3389/fphar.2018.01251.

20. Chen LL, Lei LH, Ding PH, Tang Q, Wu YM. Osteogenic effect of Drynariae rhizoma extracts and Naringin on MC3T3-E1 cells and an induced rat alveolar bone resorption model. Archives of oral biology. 2011;56(12):1655-62. doi:10.1016/j.archoralbio.2011.06.008.

21. Sun W, Li M, Zhang Y, Huang Y, Zhan Q, Ren Y, et al. Total flavonoids of rhizoma drynariae ameliorates bone formation and mineralization in BMP-Smad signaling pathway induced large tibial defect rats. Biomedicine \& pharmacotherapy = Biomedecine \& pharmacotherapie 2021; 138111480. doi: 10.1016/j.biopha.2021.111480.

22. Guo D, Wang J, Wang X, Luo H, Zhang H, Cao D, et al. Double directional adjusting estrogenic effect of naringin from Rhizoma drynariae (Gusuibu). J Ethnopharmacol. 2011;138(2):451-7. doi:10.1016/j.jep.2011.09.034.

23. Cheng CF, Chien-Fu Lin J, Tsai FJ, Chen CJ, Chiou JS, Chou CH, et al. Protective effects and network analysis of natural compounds obtained from Radix dipsaci, Eucommiae cortex, and Rhizoma drynariae against RANKL-induced osteoclastogenesis in vitro. J Ethnopharmacol. 2019;244:112074. doi:10.1016/j.jep.2019.112074.

24. Yang B, Lin X, Tan J, She X, Liu Y, Kuang H. Root bark of Sambucus Williamsii Hance promotes rat femoral fracture healing by the BMP-2/Runx2 signaling pathway. J Ethnopharmacol. 2016;191:10714. doi:10.1016/j.jep.2016.05.017.

25. Chen M, Han H, Zhou S, Wen Y, Chen L. Morusin induces osteogenic differentiation of bone marrow mesenchymal stem cells by canonical Wnt/ $\beta$-catenin pathway and prevents bone loss in an ovariectomized rat model. Stem Cell Res Ther. 2021;12(1):173. doi:10.1186/s13287-021-02239-3. 
26. Tang CY, Wu M, Zhao D, Edwards D, McVicar A, Luo Y, et al. Runx1 is a central regulator of osteogenesis for bone homeostasis by orchestrating BMP and WNT signaling pathways. PLoS Genet. 2021;17(1):e1009233. doi:10.1371/journal.pgen.1009233.

27. Song J, Jiang N, Gan X, Zhi W, Zhu Z. Thrombin inhibitor argatroban modulates bone marrow stromal cells behaviors and promotes osteogenesis through canonical Wnt signaling. Life sciences 2021; 269 119073. doi: 10.1016/j.Ifs.2021.119073.

28. Shen G, Ren H, Shang Q, Zhao W, Zhang Z, Yu X, et al. Foxf1 knockdown promotes BMSC osteogenesis in part by activating the $\mathrm{Wnt} / \beta$-catenin signalling pathway and prevents ovariectomyinduced bone loss. EBioMedicine. 2020;52:102626. doi:10.1016/j.ebiom.2020.102626.

29. Jeong JC, Lee JW, Yoon CH, Kim HM, Kim CH. Drynariae Rhizoma promotes osteoblast differentiation and mineralization in MС3T3-E1 cells through regulation of bone morphogenetic protein-2, alkaline phosphatase, type I collagen and collagenase-1. Toxicology in vitro: an international journal published in association with BIBRA 2004; 18 (6): 829-834. doi: 10.1016/j.tiv.2004.05.002.

30. Gan D, Xu X, Chen D, Feng P, Xu Z. Network Pharmacology-Based Pharmacological Mechanism of the Chinese Medicine Rhizoma drynariae Against Osteoporosis. Medical science monitor: international medical journal of experimental clinical research. 2019;25:5700-16. doi:10.12659/msm.915170.

31. Shi XL, Liu K, Wu LG. Interventional value of total flavonoids from Rhizoma Drynariae on Cathepsin $\mathrm{K}$, a potential target of osteoporosis. Chin J Integr Med. 2011;17(7):556-60. doi:10.1007/s11655010-0792-1.

32. Sen B, Paradise CR, Xie Z, Sankaran J, Uzer G, Styner M, et al. $\beta$-Catenin Preserves the Stem State of Murine Bone Marrow Stromal Cells Through Activation of EZH2. Journal of bone mineral research: the official journal of the American Society for Bone Mineral Research. 2020;35(6):1149-62. doi:10.1002/jbmr.3975.

\section{Figures}
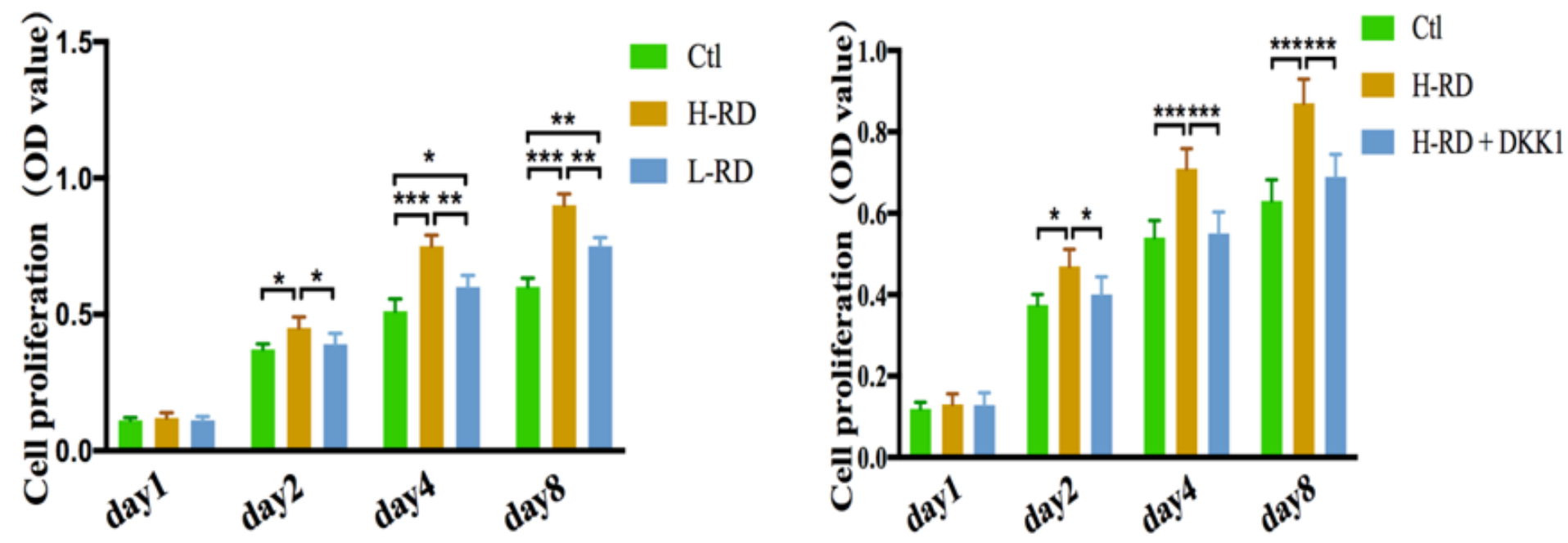
Figure 1

MTT assay analysis of the BMSC proliferation $(n=4)$. Data are represented as the mean \pm standard deviation; ${ }^{*} P<0.05,{ }^{\star} P P<0.01,{ }^{\star \star *} P<0.001$ by one-way analysis of variance with Tukey's post hoc test. Ctl: control group; H-RD: high RD-containing serum group; L-RD: low RD-containing serum group; H-RD + DKK1: high RD-containing serum co-treated with DKK1.

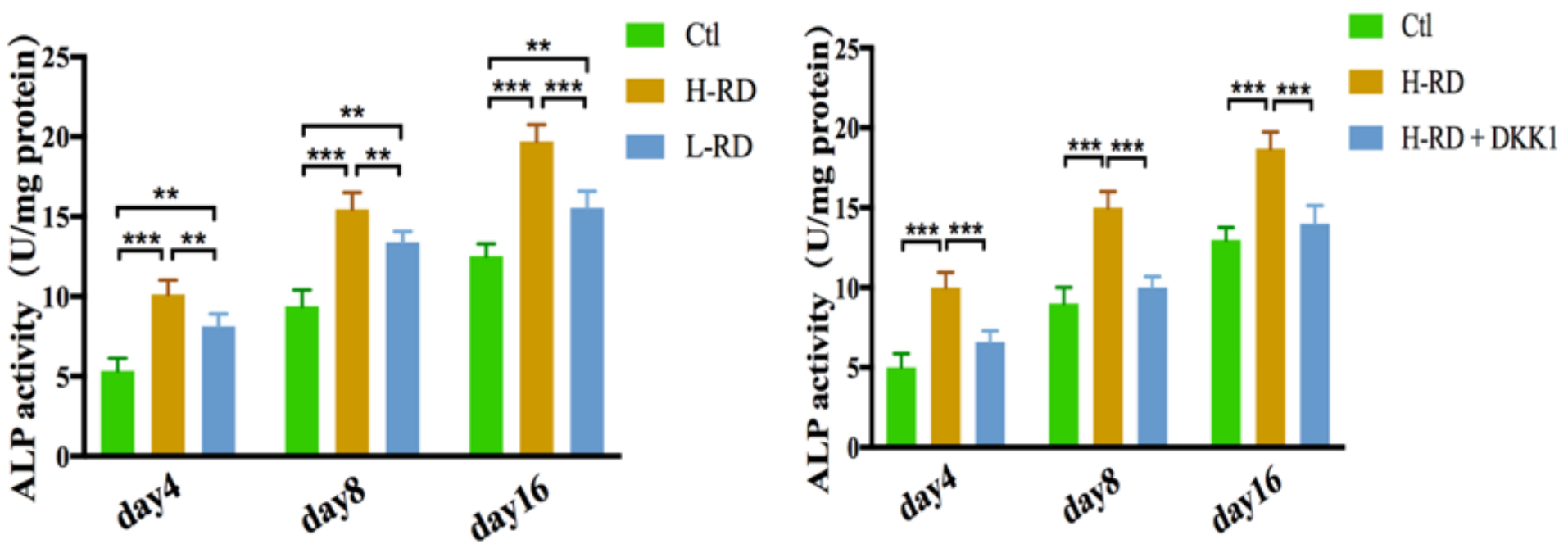

Figure 2

ALP activity assay of the three groups. BMSCs cultured in OIM co-treated with or without RD-containing serum or DKK1 as indicated $(n=4)$. The data are presented as the means \pm SD of independent experiments; ${ }^{*} P<0.05,{ }^{*} P<0.01,{ }^{* \star *} P<0.001$ by one-way analysis of variance with Tukey's post hoc test. Ctl: control group; H-RD: high RD-containing serum group; L-RD: low RD-containing serum group; $\mathrm{H}-$ RD + DKK1: high RD-containing serum co-treated with DKK1.

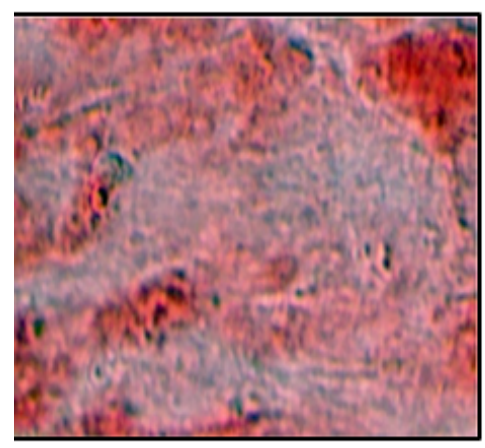

Ct1

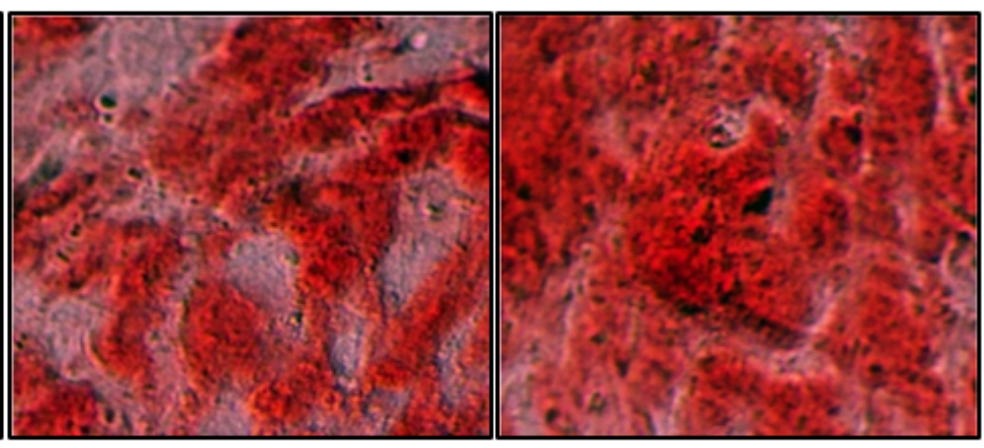

$\mathrm{L}-\mathrm{RD}$

$\mathrm{H}-\mathrm{RD}$

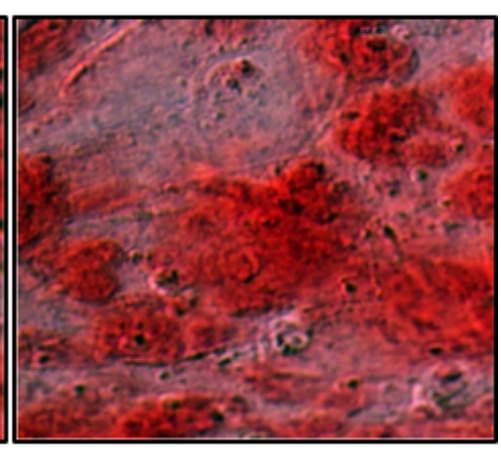

$\mathrm{H}-\mathrm{RD}+\mathrm{DKK} 1$

\section{Figure 3}

BMSCs were cultured in an OIM for 16 days, fixed and stained with Alizarin red. Low RD-containing serum and high RD-containing serum markedly increased the osteogenic differentiation of BMSCs, while DKK1 inhibited the promotion of high RD-containing serum on the osteogenic differentiation of BMSCs. Ctl: control group; H-RD: high RD-containing serum group; L-RD: low RD-containing serum group; H-RD + DKK1: high RD-containing serum co-treated with DKK1. 

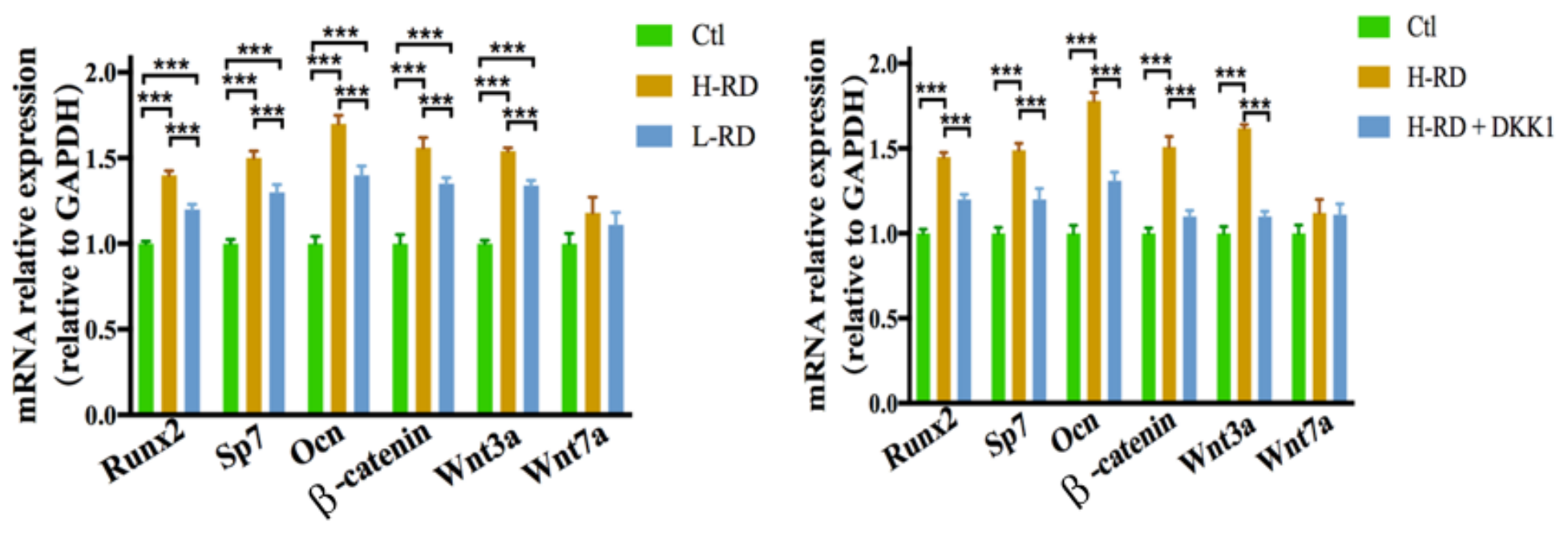

Figure 4

RT-PCR was used to examine changes in the levels of the Runx2, Sp7, Ocn, $\beta$-catenin, Wnt3a and Wnt7a mRNAs in BMSCs cultured in OIM co-treated with or without RD-containing serum or DKK1 as indicated (n $=4$ ). Data are represented as the mean \pm standard deviation; ${ }^{*} P<0.05,{ }^{\star *} P<0.01,{ }^{\star} * \star P<0.001$ by oneway analysis of variance with Tukey's post hoc test. Ctl: control group; H-RD: high RD-containing serum group; L-RD: low RD-containing serum group; H-RD + DKK1: high RD-containing serum co-treated with DKK1.

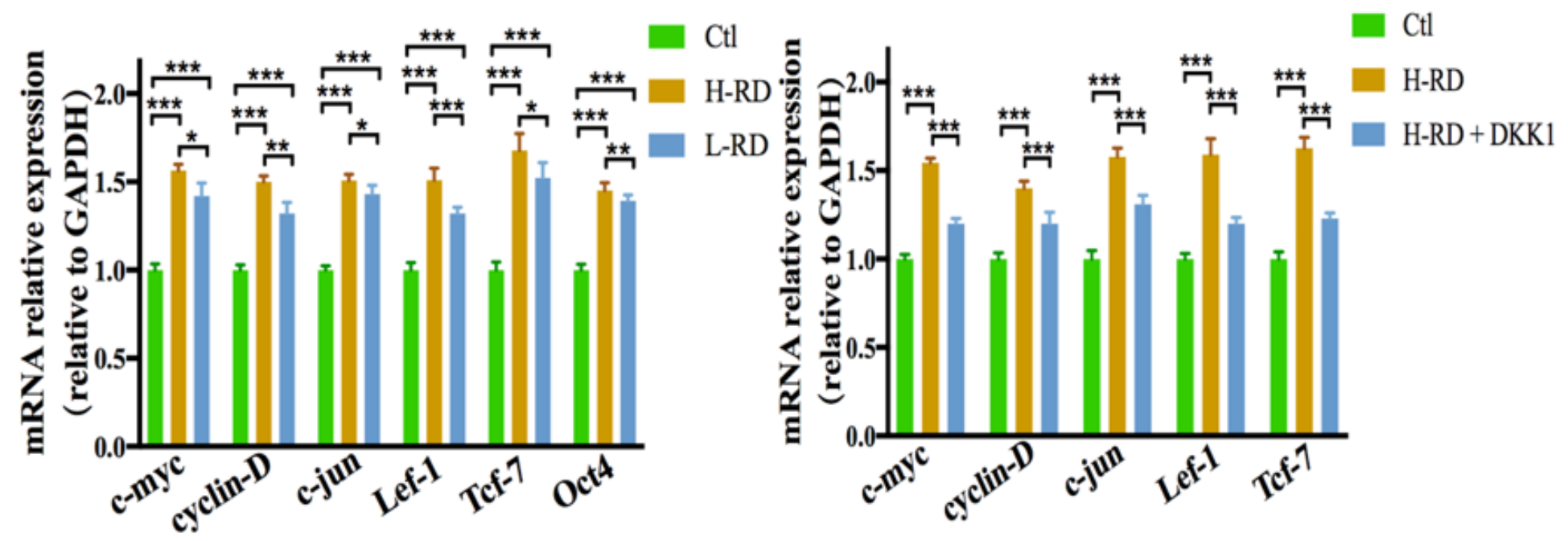

Figure 5

Effects of RD-containing serum and DKK1 on Wnt/ $\beta$-catenin related gene expression in BMSCs cultured in OIM for 16 days $(n=4)$. Data are represented as the mean \pm standard deviation; ${ }^{*} P<0.05, * \star P<0.01$, ${ }^{\star} \star * \mathrm{P}<0.001$ by one-way analysis of variance with Tukey's post hoc test. Ctl: control group; H-RD: high RDcontaining serum group; L-RD: low RD-containing serum group; H-RD + DKK1: high RD-containing serum co-treated with DKK1. 

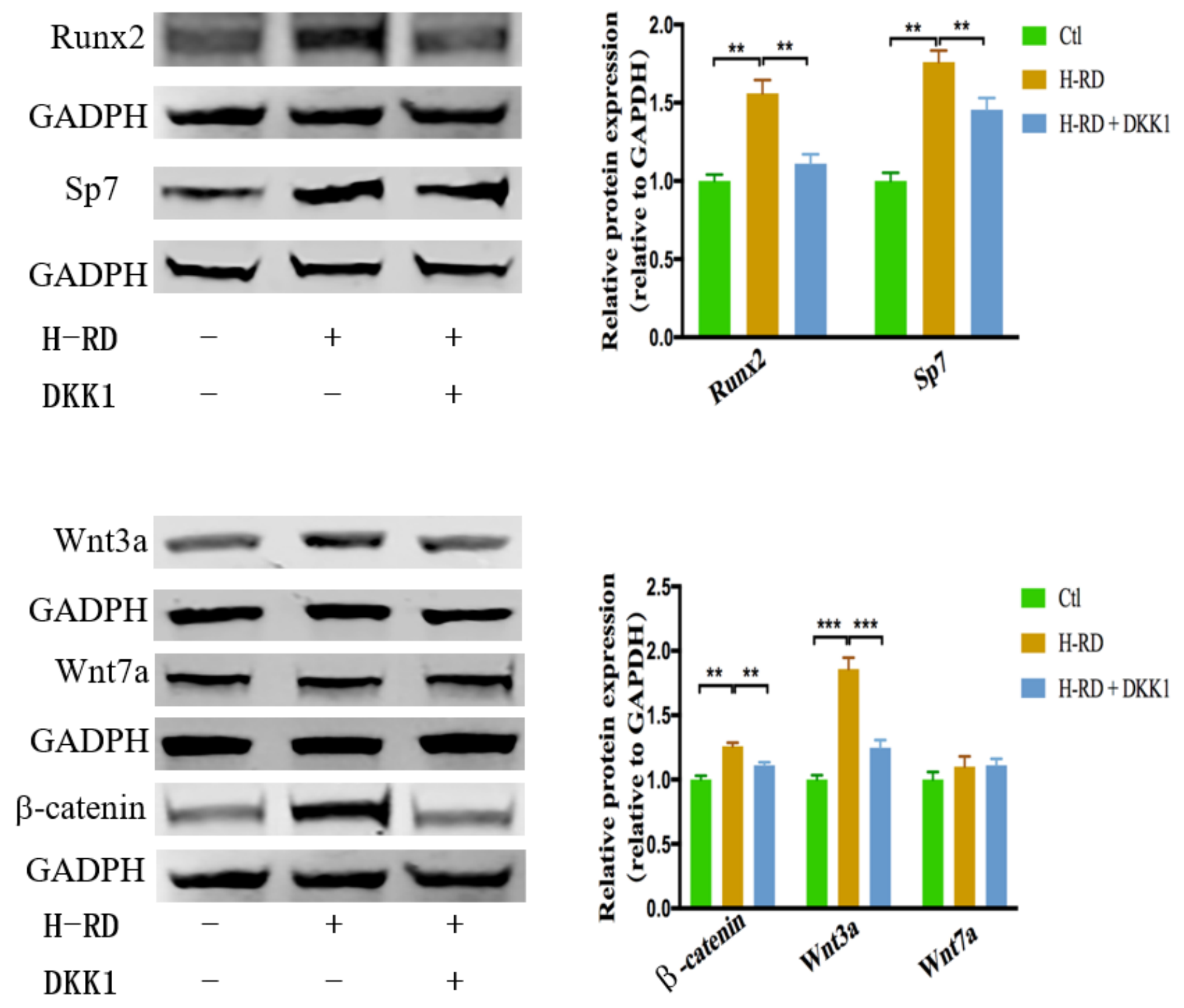

Figure 6

Effects of RD-containing serum and DKK1 on Wnt/ $\beta$-catenin protein expression in BMSCs cultured in OIM for 16 days $(n=4)$. Data are represented as the mean \pm standard deviation; ${ }^{*} P<0.05,{ }^{*} P<0.01, * \star * P<$ 0.001 by one-way analysis of variance with Tukey's post hoc test. Ctl: control group; H-RD: high RDcontaining serum group; H-RD + DKK1: high RD-containing serum co-treated with DKK1. 


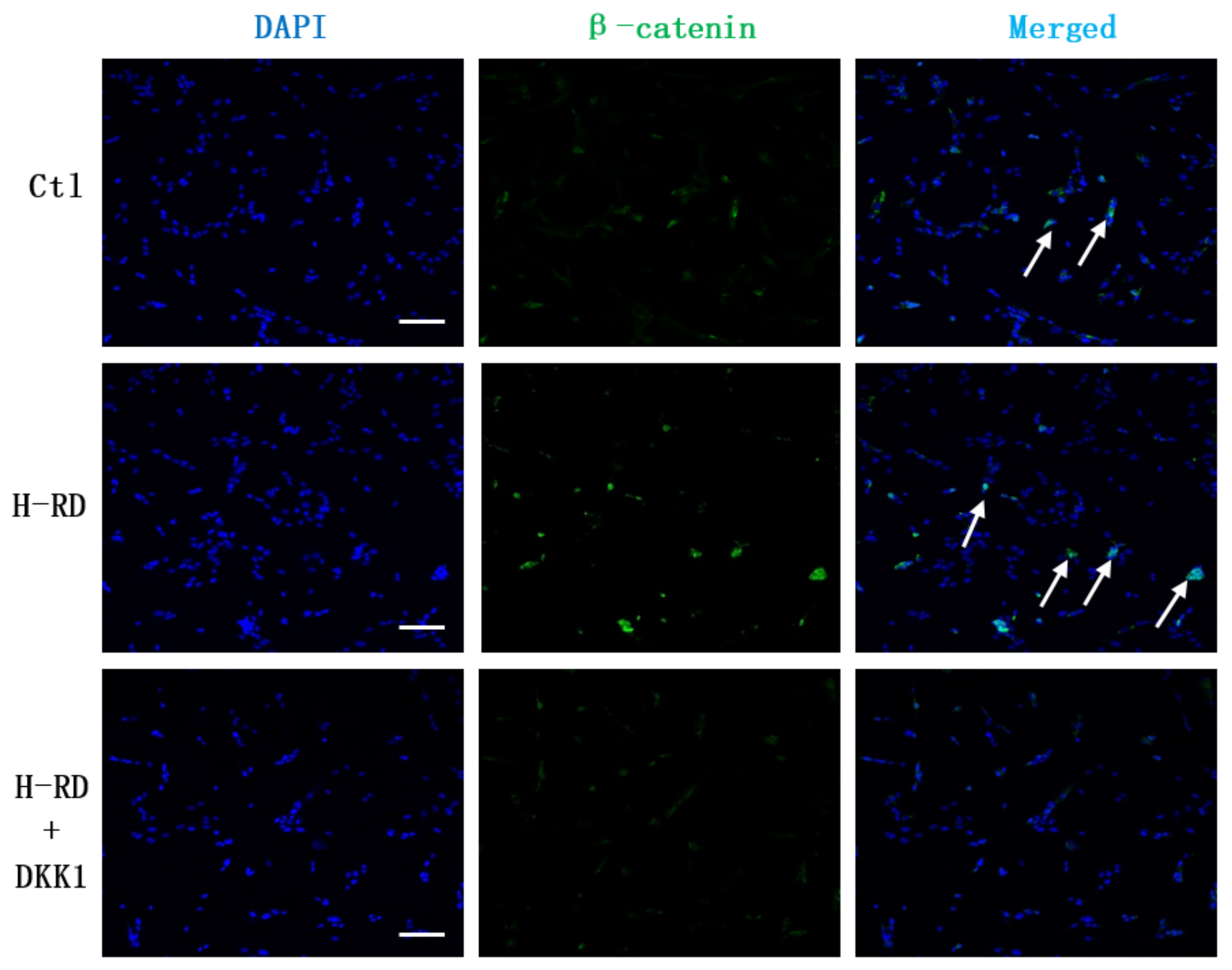

Figure 7

Effects of RD-containing serum and DKK1 on the nuclear translocation of $\beta$-catenin after adding the osteogenic induction culture to BMSCs. Representative images of the immunofluorescence staining for $\beta$ catenin (green) and DAPI (a nuclear stain, blue) in BMSCs after a 16day osteogenic incubation. White arrow: nuclear localization of $\beta$-catenin. Scale bar, $250 \mu \mathrm{m}$. Ctl: control group; H-RD: high RD-containing serum group; H-RD + DKK1: high RD-containing serum co-treated with DKK1.

\section{Supplementary Files}

This is a list of supplementary files associated with this preprint. Click to download.

- Graphicabstract.jpg 